\title{
Avaliação das propriedades dinâmico mecânicas e reológicas de compositos nanoestruturados de PPS/MWCNT
}

\section{Evaluation of dynamic mechanical and rheological properties of MWCNT/PPS nanostructured composites}

\author{
Bruno Ribeiro ${ }^{1 *}$, Edson Cocchieri Botelho ${ }^{1}$, Michelle Leali Costa ${ }^{1}$ e Cirlene Fourquet Bandeira ${ }^{1}$ \\ 'Departamento de Materiais e Tecnologia, Universidade Estadual Paulista "Júlio de Mesquita Filho" - \\ UNESP, Guaratinguetá, SP, Brazil \\ *dorado.bruno@gmail.com
}

\begin{abstract}
Resumo
Neste estudo, foram avaliadas as propriedades dinâmico mecânicas e a viscosidade de compósitos nanoestruturados de poli(sulfeto de fenileno) (PPS) reforçado com nanotubos de carbono de paredes múltiplas (MWCNT) obtidos através da técnica de mistura em fusão. O módulo de armazenamento (E') e a temperatura de transição vítrea ( $\mathrm{T}_{\mathrm{g}}$ ) apresentaram aumentos de até $130 \%$ e $11{ }^{\circ} \mathrm{C}$ em relação ao PPS puro quando $2,0 \mathrm{~m} / \mathrm{m} \%$ do nanoreforço foi considerado. Além disso, para concentrações superiores a $1,0 \%$ em massa foi observado um aumento nos valores da viscosidade complexa $\left(\eta^{*}\right)$, o que sugere a formação de uma estrutura interconectada de nanotubos no interior da matriz polimérica.
\end{abstract}

Palavras-chave: $M W C N T$, PPS, propriedades dinâmico mecânicas, viscosidade complexa.

\section{Abstract}

In this work, dynamic mechanical properties and viscosity of multiwalled carbon nanotubes reinforced poly (phenylene sulfide) nanostructured composites obtained by melt mixing techinique were investigated. The storage modulus (E') and the glass transition temperature $\left(\mathrm{T}_{\mathrm{g}}\right)$ showed increases up to $130 \%$ and $11{ }^{\circ} \mathrm{C}$ for concentrations of $2.0 \mathrm{wt} \%$ compared to neat PPS. In Addition, it was observed for concentrations above $1.0 \mathrm{wt} \%$ an increment of complex viscosity $(\eta *)$, suggesting the formation of an interconnected nanotube structure within the polymeric matrix.

Keywords: MWCNT, PPS, dynamic mechanical properties, complex viscosity.

\section{Introdução}

A nanotecnologia pode ser definida como a ciência que descreve a criação, a manipulação e a exploração de materiais em escala nanométrica. A alta demanda para criação de materiais multifuncionais de alta qualidade vem desenvolvendo uma necessidade na comunidade cientifica em formular e preparar os chamados materiais nanoestruturados. Dentre estes, pode-se citar as nanopartículas, os nanocristais, os nanofios, as nanofitas, os nanotubos e os compósitos nanoestruturados ${ }^{[1,2]}$.

Compósitos poliméricos nanoestruturados podem ser definidos por materiais híbridos orgânicos e inorgânicos, onde a fase inorgânica encontra-se dispersa em nível nanométrico em uma matriz polimérica ${ }^{[3]}$. Atualmente a busca dos nanomateriais encontra-se na descoberta de métodos de preparação, caracterização e manipulação dessas nanoestruturas para um uso específico, como no caso da nanotecnologia associada aos materiais compósitos.
Diante disso, os nanotubos de carbono aparecem como um dos materiais mais promissores associados aos compósitos poliméricos.

Os nanotubos de carbono vem ganhando grande destaque na comunidade científica, desde que foram descobertos por Iijima em $1991^{[4]}$. Dentre suas mais notáveis propriedades destaca-se a elevada resistência mecânica e a excelente condutividade elétrica e térmica, fazendo deste material um potencial candidato a nanoreforço na preparação de compósitos poliméricos nanoestruturados. No entanto, um dos obstáculos para o uso em escala industrial dessas nanoestruturas está no elevado custo dos nanotubos de carbono quando comparado ao valor da matriz polimérica, ou mesmo a outros tipos de reforços. Além disso, a otimização do processo de dispersão dos nanotubos na matriz polimérica ainda é o principal desafio para produção em larga escala dos materiais nanoestruturados ${ }^{[5-11]}$. 
Os polímeros termoplásticos apresentam um grande campo de aplicação em diversos setores da indústria, tais como: automotiva, eletrônica, e aeroespacial. Dentre suas principais vantagens pode-se citar sua baixa densidade, baixo custo e facilidade na obtenção de geometrias complexas, levando a um menor custo de processamento ${ }^{[12-14]}$. Dentro deste conceito, o poli (sulfeto de fenileno) surge como um dos principais candidatos a matriz polimérica na obtenção de compósitos poliméricos nanoestruturados. Dentre suas principais propriedades pode-se destacar sua elevada estabilidade térmica, boa resistência química e excelentes propriedades de fricção, sendo o mesmo amplamente utilizado em aplicações automotivas, eletrônicas e químicas ${ }^{[15-17]}$. Além disso, o PPS é um polímero aromático que apresenta anéis benzênicos em sua estrutura e portanto, uma forte interação entre matriz e nanoreforço é esperada, fornecendo assim, a viabilidade necessária para explorar os compósitos de PPS/MWCNT. Trabalhos anteriores já publicados nesta revista ${ }^{[18,19]}$, avaliaram o processo de dispersão do nanoreforço na matriz PPS, bem como estudos envolvendo as propriedades térmicas (degradação e cristalização) e elétricas do sistema nanoestruturado foram realizados. Portanto, o presente trabalho trata-se de uma continuação da pesquisa anteriormente apresentada, visando a caracterização de compósitos nanoestruturados de PPS/MWCNT.

O objetivo deste trabalho é avaliar as propriedades dinâmico mecânicas de compósitos nanoestruturados de poli (sulfeto de fenileno) reforçados com nanotubos de carbono. Além disso, o material foi submetido a ensaios reológicos com o objetivo de determinar a influência da adição de reforços nanoestruturados na viscosidade da matriz empregada.

\section{Materiais e Métodos}

\subsection{Materiais utilizados}

O poli(sulfeto de fenileno) (PPS) foi fornecido pela empresa Ticona (sob o nome comercial de Fortron 0205P4) na forma de grânulos. Os nanotubos de carbono de paredes múltiplas (MWCNTs), utilizados neste trabalho, foram produzidos pela técnica de deposição química de vapor (CVD) e fornecidos pela empresa Bayer, codificado como Baytubes C 150 P. Estes são caracterizados por apresentarem um diâmetro médio de suas paredes entre 13-16 nm, número de paredes entre 3-15 e massa específica entre $140-160 \mathrm{~kg} / \mathrm{m}^{3}$.

\subsection{Obtenção dos compósitos nanoestruturados de PPS/MWCNT}

Os compósitos nanoestruturados de PPS/MWCNT foram processados por meio da técnica de mistura em fusão em uma micro extrusora de rosca dupla (DSM Xplore), a $300{ }^{\circ} \mathrm{C}$, velocidade de rotação de $300 \mathrm{rpm}$ e tempo de residência de 1 minuto. Ao fim do processo, amostras com $0,5,1,0,2,0,4,0$ e 8,0\% em massa de MWCNT foram obtidas. Com o intuito de ter um melhor entendimento das propriedades reológicas, amostras com 3,0 m/m\% de MWCNT foram preparadas. Amostras de PPS foram preparadas utilizando o mesmo procedimento descrito acima com o objetivo de padronizar os ensaios de DMA e reológicos. Após a extrusão todas as amostras foram secas em uma estufa a vácuo a $120^{\circ} \mathrm{C}$ por 3 horas.

Após o processamento por fusão, os compósitos obtidos foram moldados por compressão utilizando uma prensa hidráulica (Carver Hot Press, modelo 3725), a temperatura de $300{ }^{\circ} \mathrm{C}$ por $1 \mathrm{~min}$, a uma pressão de $10 \mathrm{MPa}$. Foram obtidos filmes com geometria retangular e espessura de aproximadamente $0,5 \mathrm{~mm}$, visando a caracterização mecânica e reológica do material.

\subsection{Análise dinâmico mecânica (DMA)}

As análises de DMA foram realizadas em um equipamento da TA Instruments Dynamic Mechanical Analyzer modelo Q800. As amostras foram cortadas nas dimensões de aproximadamente, $(19,5 \times 4 \times 0,5) \mathrm{mm}$ e conduzidas em um equipamento operando no modo de flexão (single cantilever), frequência de $1 \mathrm{~Hz}$, faixa de temperatura de 40 a $200{ }^{\circ} \mathrm{C}$, com taxa de aquecimento de $2{ }^{\circ} \mathrm{C} / \mathrm{min}$, força de $6 \mathrm{~N}$ e amplitude de oscilação de $30 \mu \mathrm{m}$.

\subsection{Propriedades reológicas}

A caracterização reológica dos compósitos nanoestruturados foi realizada em um reômetro de deformação controlada da TA Instruments ARES, modelo LS2, de placas paralelas. As análises reológicas dos compósitos nanoestruturados obtidos foram realizadas a partir de ensaios de cisalhamento oscilatório de pequenas amplitudes (COPA), a temperatura $300{ }^{\circ} \mathrm{C}$, amplitude de tensão de $1 \%$, em intervalos de $0,1 \mathrm{a} 100 \mathrm{rad} / \mathrm{s}$ de frequência e em atmosfera de nitrogênio.

\section{Resultados e Discussões}

\subsection{Análise de DMA de compósitos nanoestruturados de PPS/MWCNT}

O comportamento viscoelástico dos compósitos nanoestruturados obtidos foram avaliados a partir da análise dinâmico mecânica. A técnica é útil em detectar variações na rigidez dos compósitos nanoestruturados em função da temperatura, além de ser sensível a diversos processos de relaxação e transições da matriz no compósito. Além disso, fornece informações importantes sobre a interface reforço-matriz ${ }^{[20,21]}$. A Figura 1 apresenta os principais resultados quanto à variação do módulo de armazenamento (E') em função da temperatura para o PPS puro e para seus compósitos nanoestruturados PPS/MWCNT. Como pode ser observado, o valor de E' para o PPS diminui lentamente com o aumento da temperatura, apresentando um grande decaimento na faixa de temperatura entre $80{ }^{\circ} \mathrm{C}-110^{\circ} \mathrm{C}$. Este intervalo de temperatura encontra-se associado à temperatura de transição vítrea $\left(\mathrm{T}_{\mathrm{g}}\right)$ da matriz polimérica.

Como observado a partir da Figura 1, a incorporação de até 2,0\% em massa de MWCNT foi responsável por um aumento contínuo no valor do módulo de armazenamento da matriz polimérica. Em comparação ao PPS puro, as amostras com 1,0 e 2,0\% em massa de MWCNT apresentaram aumentos de 75 e 130\% no valor de E', respectivamente. De acordo com trabalhos recentemente publicados nesta revista ${ }^{[18]}$, o aumento no módulo de armazenamento das amostras reforçadas com nanotubos de carbono pode ser explicado 


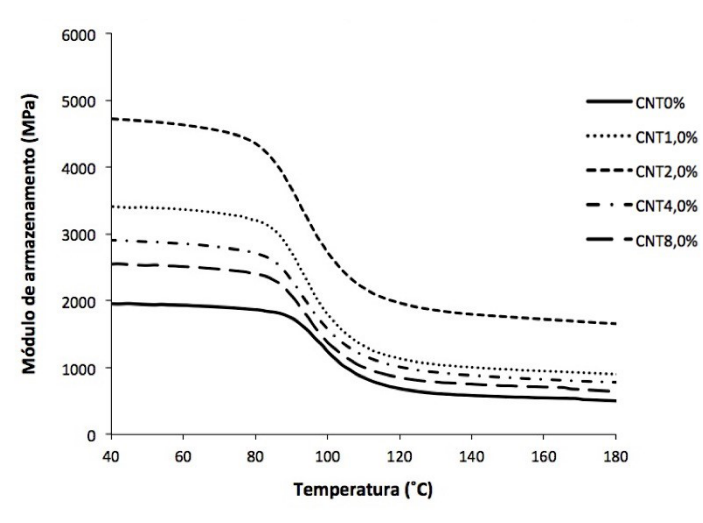

Figura 1. Módulo de armazenamento em função da temperatura para compósitos nanoestruturados de PPS/MWCNT.

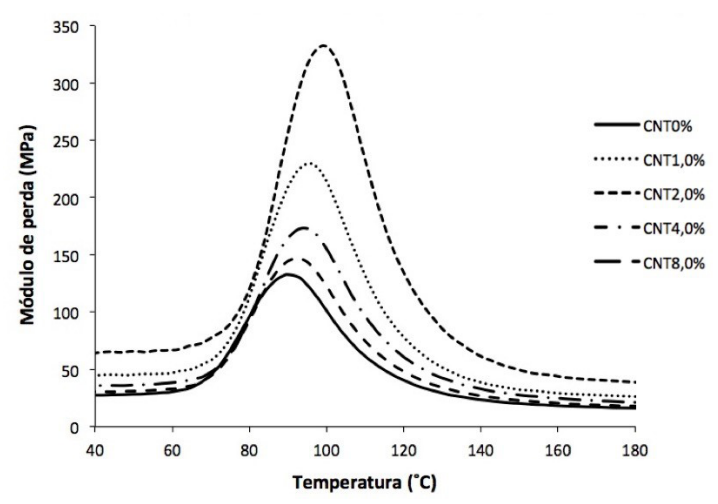

Figura 2. Módulo de perda em função da temperatura para compósitos nanoestruturados de PPS/MWCNT.

devido `a dispersão eficiente deste nanoreforço na matriz PPS. Além disso, este comportamento pode também estar associado `a restrição da mobilidade dos grupos laterais devido ao impedimento estérico promovido pelas interações entre a matriz polimerica e o nanoreforço, promovendo assim, o aumento nos valores de $\mathrm{E}^{,[22]}$. Por outro lado, para elevadas concentrações do reforço nanoestruturado (4,0 e 8,0\%) uma redução no módulo de armazenamento foi observada, de modo que os aumentos em relação a matriz polimérica foram de 42 e $25 \%$, respectivamente. Conforme apresentado anteriormente ${ }^{[23]}$, o aumento da concentração de MWCNT durante o processamento dos compósitos nanoestruturados está diretamente associado com o aumento da viscosidade do sistema. Este fato dificulta o processo de dispersão do nanoreforço no interior da matriz polimérica, reduzindo o efeito dos MWCNT como reforço, limitando assim o valor obtido do módulo de armazenamento para as amostras estudadas.

A Figura 2 apresenta a dependência da temperatura no módulo de perda (E”) do PPS puro e dos compósitos nanoestruturados de PPS/MWCNT. Como pode ser observado, a curva para o PPS apresenta um pico na temperatura de $90^{\circ} \mathrm{C}$, o que caracteriza a temperatura de transição vítrea do material. Como pode ser observado, a adição de MWCNT na matriz polimérica foi responsável por um aumento na $T_{g}$ do PPS, conforme apresentado na Tabela 1. De maneira similar
Tabela 1. Temperatura de transição vítrea dos compósitos nanoestruturados de PPS/MWCNT.

\begin{tabular}{cc}
\hline Amostras & $\mathbf{T}_{\mathbf{g}}\left({ }^{\circ} \mathbf{C}\right)$ \\
\hline CNT0\% & 90,1 \\
CNT1,0\% & 95,9 \\
CNT2,0\% & 101,5 \\
CNT4,0\% & 94,6 \\
CNT, $0 \%$ & 93,2 \\
\hline
\end{tabular}

ao módulo de armazenamento, a temperatura de transição vítrea apresenta um aumento máximo quando $2,0 \%$ em massa do nanoreforço é considerado (aproximadamente $11^{\circ} \mathrm{C} \mathrm{em}$ relação ao PPS puro). Por outro lado, para concentrações superiores a 2,0\% em massa (neste caso, 4,0 e 8,0\%) uma pequena redução nos valores da $\mathrm{T}_{\mathrm{g}}$ é observada. Conforme apresentado na literatura ${ }^{[24]}$, a incorporação de MWCNT na matriz PPS restringe a mobilidade das cadeias poliméricas, resultando no aumento deste parâmetro. Para as amostras com 4,0 e 8,0\% em massa do material nanoestruturado a redução nos valores da $T_{g}$ encontra-se provavelmente associada ao processo de dispersão pobre dos nanotubos na matriz PPS. Resultados similares para os valores de transição vítrea do sistema PPS/MWCNT foram apresentados por Yang e colaboradores $^{[25]}$.

\subsection{Análise reológica}

A importância do estudo das propriedades reológicas de compósitos poliméricos reforçados com nanotubos de carbono está associada a determinação das condições de processamento dessas nanoestruturas, além de fornecer um melhor conhecimento das características dos materiais em escala nanométrica. Além disso, a melhora no comportamento mecânico de polímeros reforçados com nanotubos de carbono pode ser explicada devido a formação de uma microestrutura gerada pelo nanoreforço no interior da matriz polimérica. A formação de tal microestrutura pode ser investigada no estado líquido a partir de experimentos reológicos ${ }^{[26]}$.

A viscosidade complexa é considerada um importante parâmetro na caracterização das propriedades reológicas de materiais poliméricos. A Figura 3 apresenta a viscosidade complexa $\left(\eta^{*}\right)$ em função da frequência de oscilação para o PPS puro e para os compósitos nanoestruturados PPS/MWCNT. Como pode ser observado, a viscosidade da matriz polimérica é independente da frequência de oscilação, apresentando um comportamento tipicamente Newtoniano. Além disso, foi observado que a incorporação do nanoreforço em concentrações de até 1,0\% em massa não altera de maneira significativa os valores da viscosidade do PPS, uma vez que apenas aumentos modestos foram observados para este parâmetro. Por outro lado, para concentrações superiores a 1,0\% em massa de MWCNT, um aumento significativo na viscosidade dos compósitos nanoestruturados a baixas frequências é apresentado. De acordo com literatura ${ }^{[27,28]}$, as interações entre as partículas de reforço em compósitos poliméricos nanoestruturados são responsáveis por um aumento da viscosidade do sistema, de modo a resultar no desaparecimento do "platô", que é característico de sistemas Newtonianos. Além disso, mudanças repentinas na viscosidade indicam que a concentração de nanoreforços 


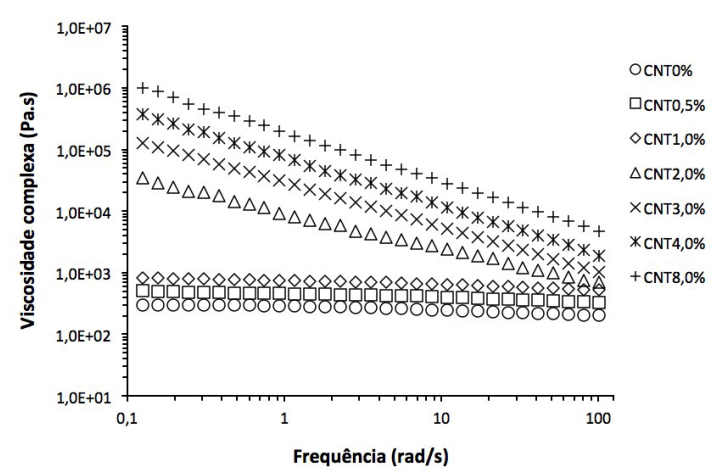

Figura 3. Viscosidade complexa em função da frequência para compósitos nanoestruturados de PPS/MWCNT.

nos compósitos nanoestruturados atinge um limite, uma vez que os mesmos impedem o movimento das cadeias do polímero, formando uma estrutura em rede no interior da matriz polimérica. Este fato sugere uma transição de comportamento do material, evidenciando o comportamento pseudoplástico obtido pelas amostras quando concentrações superiores a $1,0 \%$ em massa do nanoreforço foi considerado. Estudos anteriores apontam que a transição para o comportamento pseudoplástico do sistema nanoestruturado de PPS/MWCNT ocorreu em concentrações ligeiramente superiores ao reportado neste trabalho. Yang et al. ${ }^{[25]}$ e Han et al. ${ }^{[29]}$, determinaram, respectivamente, concentrações superiores a $2,0 \mathrm{~m} / \mathrm{m} \%$ e $3,0 \mathrm{~m} / \mathrm{m} \%$. A partir dos dados citados acima, fica claro que o procedimento experimetal realizado e a escolha da técnica de mistura em fusão foram adequados para a obtenção dos compósitos de PPS/MWCNT, promovendo assim, a formação de uma rede tridimensional no interior da matriz polimérica em concentrações inferiores de nanoreforço.

\section{Conclusões}

A incorporação dos MWCNT no PPS foi responsável pelo aumento de até $130 \%$ no módulo de armazenamento dos compósitos de PPS/MWCNT em relação ao PPS puro quando $2,0 \mathrm{~m} / \mathrm{m} \%$ do nanoreforço foi considerado. Além disso, incrementos de até $11^{\circ} \mathrm{C}$ na temperatura de transição vítrea foram observados quando 2,0\% em massa do nanoreforço foi adicionado a matriz polimérica.

A viscosidade do PPS também foi afetada pela presença dos MWCNT. Concentrações superiores a 1,0\% em massa do nanoreforço sugerem a formação de uma estrutura em rede no interior da matriz polimérica, evidenciando o comportamento pseudoplástico adquirido pelas amostras.

\section{Agradecimentos}

Os autores agradecem a FAPESP (projeto 2008/00171-8), ao CNPq (474160/2010-6 e 303559/2012-8) e a CAPES (18910-12-2) pelo suporte financeiro, sem os quais a realização deste trabalho não seria possível.

\section{Referências}

1. Khan, M. O., Leung, S. N., Chan, E., Naguib, H. E., Dawson, F., \& Adinkrah, V. (2013). Effects of microsized and nanosized carbon fillers on the thermal and electrical properties of polyphenylene sulfide based composites. Polymer Engineering and Science, 53(11), 2398-2406. http://dx.doi.org/10.1002/ pen.23503.

2. Pereira, M. B.(2003). Caracterização de filmes ópticos compósitos nanoestruturados, inomogeneos ou anisotrópicos, produzidos por troca ionica e pelo método sol-gel (Dissertação de mestrado). Universidade Federal do Rio Grande do Sul, Porto Alegre.

3. Guimarães, T. R., Morales, A. R., \& Paiva, L. B. (2006). Propriedades mecânicas de nanocompósitos de polipropileno e montmorilonita organofílica. Polímeros: Ciência e Tecnologia, 16(2), 136-140. http://dx.doi.org/10.1590/S010414282006000200014 .

4. Iijima, S. (1991). Helical microtubules of graphitic carbon. Nature, 354(6348), 56-58. http://dx.doi.org/10.1038/354056a0.

5. Farahani, R. D., Dalir, H., Borgne, V., Gautier, L. A., Khakani, M. A. E., Lévesque, M., \& Therriault, D. (2012). Reinforcing epoxy nanocomposites with functionalized carbon nanotubes via biotin-streptavidin interactions. Composites Science and Technology, 72(12), 1387-1395. http://dx.doi.org/10.1016/j. compscitech.2012.05.010.

6. Ma, P. C., Siddiqui, N. A., Marom, G., \& Kim, J. K. (2010). Dispersion and functionalization of carbon nanotubes for polymer-based nanocomposites: a review. Composites Part A, Applied Science and Manufacturing, 41(10), 1345-1367. http://dx.doi.org/10.1016/j.compositesa.2010.07.003.

7. Sun, G., Chen, G., Liu, Z., \& Chen, M. (2010). Preparation, crystallization, electrical conductivity and thermal stability of syndiotactic polystyrene/carbon nanotube composites. Carbon, 48(5), 1434-1440. http://dx.doi.org/10.1016/j. carbon.2009.12.037.

8. Tang, X. G., Hou, M., Zou, J., Truss, R., Yang, M., \& Zhu, Z. (2012). Toughening and reinforcement of poly(vinylidene fluoride) nanocomposites with "bud-branched" nanotubes. Composites Science and Technology, 2(72), 263-268. http:// dx.doi.org/10.1016/j.compscitech.2011.11.011.

9. Liu, Z., Bai, G., Huang, Y., Ma, Y., Feng, D., Li, F., Guo, T., $\&$ Chen, Y. (2007). Reflection and absorption contributions to the electromagnetic interference shielding of single-walled carbon nanotube/polyurethane composites. Carbon, 45(4), 821-827. http://dx.doi.org/10.1016/j.carbon.2006.11.020.

10. Vidhate, S., Innocentini-Mei, L., \& D'Souza, N. A. (2012). Mechanical and electrical multifunctional poly(3hydroxybutyrate-co-3-hydroxyvalerate): multiwall carbon nanotube nanocomposites. Polymer Engineering and Science, 52(6), 1367-1374. http://dx.doi.org/10.1002/pen.23084.

11. Ribeiro, B., Botelho, E. C., \& Costa, M. L. (2014). Estudo da cinética de decomposição de compósitos nanoestruturados de poli (Sulfeto de Fenileno) reforçados com nanotubos de carbono. Polímeros: Ciência e Tecnologia, 24(6), 720-725. http://dx.doi.org/10.1590/0104-1428.1698.

12. Díez-Pascual, A. M., Naffakh, M., Marco, C., \& Ellis, G. (2012). Mechanical and electrical properties of carbon nanotube/poly(phenylene sulphide) composites incorporating polyetherimide and inorganic fullerene-like nanoparticles. Composites Part A, Applied Science and Manufacturing, 43(4), 603-612. http://dx.doi.org/10.1016/j.compositesa.2011.12.026.

13. Goyal, R. K., Kambale, K. R., Nene, S. S., Selukar, B. S., Arbuj, S., \& Mulik, U. P. (2011). Fabrication, thermal and electrical properties of polyphenylene sulphide/copper composites. 
Materials Chemistry and Physics, 128(1-2), 114-120. http:// dx.doi.org/10.1016/j.matchemphys.2011.02.065.

14. Zhou, S., Zhang, Q., Wu, C., \& Huang, J. (2013). Effect of carbon fiber reinforcement on the mechanical and tribological properties of polyamide6/polyphenylene sulfide composites. Materials \& Design, 44, 493-499. http://dx.doi.org/10.1016/j. matdes.2012.08.029.

15. Bo, Y., Long, C., Meifang, Z., \& Yanmo, C. (2007). Reactive blends of poly(phenylene sulfide)/hyperbranched poly(phenylene sulfide). Macromolecular Symposia, 254(1), 167-172. http:// dx.doi.org/10.1002/masy.200750826.

16. Chen, Z., Li, T., Yang, Y., Liu, X., \& Lv, R. (2004). Mechanical and tribological properties of PA/PPS blends. Wear, 257(7-8), 696-707. http://dx.doi.org/10.1016/j.wear.2004.03.013.

17. Noll, A., \& Burkhart, T. (2011). Morphological characterization and modelling of electrical conductivity of multi-walled carbon nanotube/poly(p-phenylene sulfide) nanocomposites obtained by twin screw extrusion. Composites Science and Technology, 71(4), 499-505. http://dx.doi.org/10.1016/j. compscitech.2010.12.026.

18. Ribeiro, B., Botelho, E. C., \& Costa, M. L. (2014). Estudo da cinética de decomposição de compósitos nanoestruturados de poli (Sulfeto de Fenileno) reforçados com nanotubos de carbono. Polimeros: Ciência e Tecnologia, 24(6), 720-725. http://dx.doi.org/10.1590/0104-1428.1698.

19. Ribeiro, B., Botelho, E. C., \& Costa, M. L. (2015). Estudo das propriedades elétricas e térmicas de compósitos nanoestruturados de poli(sulfeto de fenileno) reforçados com nanotubos de carbono. Polímeros: Ciência e Tecnologia, 25(1), 94-100. http://dx.doi.org/10.1590/0104-1428.1728.

20. Cassu, S. N., \& Felisberti, M. I. (2005). Comportamento dinâmico-mecânico e relaxações em polímeros e blendas poliméricas. Quimica Nova, 28(2), 250-254. http://dx.doi. org/10.1590/S0100-40422005000200017.

21. Kaleemullah, M., Khan, S. U., \& Kim, J. K. (2012). Effect of surfactant treatment on thermal stability and mechanical properties of CNT/polybenzoxazine nanocomposites. Composites Science and Technology, 72(16), 1968-1976. http://dx.doi. org/10.1016/j.compscitech.2012.08.020.

22. Díez-Pascual, A. M., \& Naffakh, M. (2013). Enhancing the thermomechanical behaviour of poly(phenylene sulphide) based composites via incorporation of covalently grafted carbon nanotubes. Composites. Part A, Applied Science and Manufacturing, 54, 10-19. http://dx.doi.org/10.1016/j. compositesa.2013.06.018.

23. Ribeiro, B. (2015). Obtenção e caracterização de compósitos nanoestruturados de poli (sulfeto de fenileno) reforçados com nanotubos de carbono (Tese de doutorado). Universidade Estadual Paulista, Guaratinguetá.

24. Chen, W., Lu, H., \& Nutt, S. R. (2008). The influence of functionalized MWCNT reinforcement on the thermomechanical properties and morphology of epoxy nanocomposites. Composites Science and Technology, 68(12), 2535-2542. http://dx.doi. org/10.1016/j.compscitech.2008.05.011.

25. Yang, J., Xu, T., Lu, A., Zhang, Q., Tan, H., \& Fu, Q. (2009). Preparation and properties of poly (p-phenylene sulfide)/ multiwall carbon nanotube composites obtained by melt compounding. Composites Science and Technology, 69(2), 147-153. http://dx.doi.org/10.1016/j.compscitech.2008.08.030.

26. Du, B., \& Fang, Z. (2011). Effects of carbon nanotubes on the thermal stability and flame retardancy of intumescent flame-retarded polypropylene. Polymer Degradation \& Stability, 96(10), 1725-1731. http://dx.doi.org/10.1016/j. polymdegradstab.2011.08.002.

27. Botelho, E. C., Costa, M. L., Braga, C. I., Burkhart, T., \& Lauke, B. (2013). Viscoelastic behavior of multiwalled carbon nanotubes into phenolic resin. Materials Research, 16(4), 713720. http://dx.doi.org/10.1590/S1516-14392013005000045.

28. Zhang, Q., Fang, F., Zhao, X., Li, Y., Zhu, M., \& Chen, D. (2008). Use of dynamic rheological behavior to estimate the dispersion of carbon nanotubes in carbon nanotube/polymer composites. The Journal of Physical Chemistry B, 112(40), 12606-12611. PMid:18785703. http://dx.doi.org/10.1021/ jp802708j.

29. Han, M. S., Lee, Y. K., Lee, H. S., Yun, C. H., \& Kim, W. N. (2009). Electrical, morphological and rheological properties of carbon nanotube composites with polyethylene and poly(phenylene sulfide) by melt mixing. Chemical Engineering Science, 64(22), 4649-4656. http://dx.doi.org/10.1016/j.ces.2009.02.026.

Enviado: Jun. 03, 2015

Revisado: Nov. 06, 2015 Aceito: Fev. 29, 2016 